\title{
Escala Argentina de Inteligencia Sensorio-motriz (EAIS): PERCENTILES NACIONALES*
}

\section{Argentine Scale of Sensor Motor Intelligence: National percentiles}

\author{
Alicia Oiberman $^{* *}$, Cynthia Paolini ${ }^{* * *}$ y Mariela Mansilla ${ }^{* * *}$
}

\begin{abstract}
*Trabajo realizado y financiado en el marco del proyecto Evaluación Nacional de la Inteligencia Sensorio-motriz a Bebés de 6 a 30 meses (UBACyT 2008-2010 Proyecto 034) de la Universidad de Buenos Aires (UBA).
\end{abstract}

**Doctora en Psicología. Miembro de la Carrera del Investigador del Consejo Nacional de Investigaciones Científicas y Técnicas (CONICET). Docente de la Facultad de Psicología de la Universidad de Buenos Aires (UBA).

E-Mail: aoiberma@psi.uba.ar

***Licenciada en Psicología. Becaria Doctoral del Consejo Nacional de Investigaciones Científicas y Técnicas (CONICET). Docente de la Facultad de Psicología de la Universidad de Buenos Aires (UBA).

****Licenciada en Psicología. Docente de la Facultad de Psicología de la Universidad de Buenos Aires (UBA).

Centro Interdisciplinario de Investigaciones en Psicología Matemática y Experimental "Dr. Horacio J.A. Rimoldi”. (CIIPME). Tte. Gral. Perón 2158.

(C1040AAH) Ciudad Autónoma de Buenos Aires. República Argentina.

\section{RESUMEN}

El estudio del desarrollo psicomotor de niños menores de 3 años permite observar que algunas veces, el área intelectual no es considerada en su real dimensión, como un logro específico de los niños en ese período. La Escala Argentina de Inteligencia Sensorio-motriz (EAIS) es el primer instrumento creado en Argentina para la evaluación del desarrollo cognitivo en bebés de 6 a 30 meses. Dado que no se contaba con percentiles nacionales en niños argentinos, se propuso como objetivo la realización de una validación nacional de la EAIS.

La muestra estuvo conformada por niños nacidos a término, sin patología y con un desarrollo psicomotor normal, provenientes de 10 provincias argentinas. Se conformaron grupos interdisciplinarios de trabajo en las provincias participantes para la evaluación del desarrollo cognitivo mediante la administración de la EAIS. Se logró una muestra total de 800 niños. Se construyeron tablas de percentiles nacionales para la EAIS a partir del estadío mediano obtenido en la prueba y para cada una de las series que la conforman. Los resultados permiten observar que el $72.25 \%$ de los niños obtuvieron percentiles normales, el $27.75 \%$ restante obtuvo percentiles de riesgo y retraso en la inteligencia sensorio-motriz. A partir de estos resultados se encuentra una progresión de la inteligencia gradual y cuasi-continua: ella no evoluciona más por saltos, y hacia delante, sino como una rampa con períodos de mesetas y vuelta atrás de falsos pasos.

Palabras clave: Desarrollo cognitivo; Inteligencia sensorio-motriz; Bebés; Escala argentina; Percentiles nacionales. 


\section{ABSTRACT}

The psychomotor development assessment in children younger than 3 years old allow us to realize that, sometimes, the intellectual area of development is not considered as it should be; i.e., as a specific achievement during that period of development. The Argentine Scale of Sensor Motor Intelligence (EAIS) is the first scale to assess cognitive development from Argentina. Until now we did not have national percentiles, so we decided to perform a study focusing on the national validation of the EAIS. The national validation of the Argentine Scale of Sensor motor Intelligence was performed with the aim of determining national percentiles for Argentinean children from 6 to 30 month old. The sample consisted of fullterm infants ( $>37$ weeks of gestation), without any pathology and with a normal psychomotor development. The sample was collected from 10 Argentine provinces: Buenos Aires, Córdoba, Santa Fe, Entre Ríos, Salta, Misiones, Chaco, Río Negro, Santa Cruz, and Mendoza. We organized interdisciplinary working groups in each of the ten provinces, in order to evaluate infant cognitive development in children from each Argentinean provinces through administration of the EAIS, and to perform national percentiles for the EAIS. The procedure for the realization of this work consisted in four stages: The first stage of this work consisted in the call, invitation and training to professional staff from different provinces of $\mathrm{Ar}$ gentina, to participate of the present research. A total of 30 professionals from 10 provinces decided to participate. During the second stage we proceeded to the administration of the EAIS by each of the staff of trained professionals in each of the provinces. In the third stage we achieved to collect a total sample of 800 infants from 10 Argentine provinces. Fourth stage: the realization of national percentiles tables for the EAIS and the transfer of results to scientific community is the principal aim of the present paper. We evaluated a total sample of 800 children from 6 to 30 month old. To assess the psychomotor development, the Prueba Nacional de Pesquisa (PRUNAPE) was applied to the whole sample of 800 children. Then, we evaluated the cognitive development through administration of the EAIS, to each child that had obtained a normal psychomotor development in the
PRUNAPE. We then performed national percentiles for the EAIS from the results of this analysis.

Results show us that from a total of 800 children, 578 (72.25\%) obtained normal cognitive development (percentile 50 or higher), 112 children (14\%) obtained risk percentiles (percentile 25$)$ and the 110 remaining children $(13.75 \%)$ obtained results within delay percentiles $(10$ percentile or less). In conclusion, the $27.75 \%$ of children achieved a sensor motor intelligence development below that expected in relation to the age. That percentage was similar to what we have found in previous research.

We didn't find significant differences between provinces in relation to children's cognitive development.

In previous research we found that only the $20 \%$ of 24 -month-old children had completed VI stage of sensor motor intelligence. We consider by this study an important aim was to investigate at what age Argentinean children usually complete this stage. We prove that Argentinean children usually complete VI stage of sensor motor intelligence about 30-month-old, unlike Jean Piaget's postulations. He considered that children often complete VI stage of sensor motor intelligence around the 24-month-old.

From the results, we suggest that there is a gradual and quasi-continuous progression of the intelligence, i.e., intelligence does not seem to evolve by leaps according to the ladder model instead it develops as a ramp with some retrogression and missteps.

Key words: Cognitive development; Sensor motor intelligence; Babies; Argentine scale; National percentiles.

\section{INTRODUCCIÓN}

El estudio del desarrollo psicomotor de niños menores de 3 años permite observar que algunas veces, el área intelectual no es considerada en su real dimensión, como un logro específico de los niños en ese período.

Dado que no existía en Argentina un instrumento adecuado para valorar el desarrollo 
intelectual de niños muy pequeños, la revisión de varias pruebas realizadas en otros países, llevó a elegir la Escala de Etapas de la Inteligencia de Casati y Lezine (1969) como base teórica para elaborar un instrumento estandarizado para el diagnóstico del desarrollo intelectual de bebés argentinos de 6 a 24 meses. A partir de ella hemos construido en el año 2002 la Escala Argentina de Inteligencia Sensorio-motriz (EAIS - Oiberman, Mansilla \& Orellana, 2002; Oiberman, Orellana \& Mansilla, 2006) y fue elaborada partiendo de una serie de pruebas estandarizadas basadas en las observaciones experimentales de Piaget (1973). La muestra original del año 2002 contó con una población de 323 bebés de 6 a 24 meses de las ciudades de Buenos Aires y de Avellaneda (Prov. de Buenos Aires).

Se denomina inteligencia sensorio-motriz a la capacidad de resolver problemas a partir de actividades en las que intervienen sobre todo la percepción, las actitudes, el tono muscular y los movimientos sin evocaciones simbólicas, antes de la aparición del lenguaje verbal, es decir, antes de que sea posible que el lenguaje exprese pensamiento. Pues, siguiendo el constructo piagetiano, en esta etapa la acción más que el lenguaje expresivo es fundamental para el desarrollo del pensamiento.

Después de los 2 años el pensamiento se hace lenguaje y el lenguaje se hace pensamiento. Antes de los 2 años el lenguaje no alcanza a expresar el pensamiento (Vygotsky, 1995). Dentro de este período llamado sensorio-motriz el niño poco a poco comienza a relacionar objetos y actos, distinguiendo los medios de los fines, dándose cuenta de los resultados obtenidos y utilizando hacia el fin de ese período procesos de inferencia (Oiberman et al., 2002).

La elaboración de esta escala se basó en el supuesto de que el proceso de construcción de la inteligencia sensorio-motriz constituye junto a las áreas motricidad, coordinación, lenguaje y social, la quinta área del desarrollo del niño.

Dado que la primera validación de la EAIS solo contemplaba una regionalización de los datos obtenidos, se decidió realizar un estu- dio a los efectos de elaborar percentiles nacionales para la misma. La ampliación de la edad de evaluación con esta escala hasta los 30 meses se realizó considerando que según los datos obtenidos en la primera validación en el año 2002, sólo el $20 \%$ de los niños finalizaban el período sensorio-motriz a los 24 meses como afirmaba Piaget (1973).

\section{FundamentaCión teóRICA: Conceptualizaciones SOBRE LA INTELIGENCIA}

Desde la Psicología del desarrollo, distintas conceptualizaciones sobre la inteligencia hicieron su aporte al campo de la Psicología de la infancia. Desde los precursores hasta las teorías actuales, se puede nombrar a Wallon para quien su concepto del desarrollo de la infancia resulta de la doble influencia del darwinismo y el marxismo: el medio sobre el cual se debe adaptar el niño no es solamente biológico o físico, sino también social. Wallon encara el desarrollo de la personalidad en estadíos sucesivos, desde una visión global que toma en consideración y en alternancia la afectividad y la inteligencia y afirma:

"el niño que siente va camino al niño que piensa" (1974, p. 40).

Vygotsky (1995) otorga al contexto social una importancia primordial. Tres ejes estructuran su teoría:

1.- Sostiene que es a través del intercambio con otros que el niño desarrolla su inteligencia, integrando conocimientos forjados por la historia y la cultura de la sociedad.

2.- Le otorga un lugar primordial al lenguaje ya que considera que los conocimientos son trasmitidos a través de instrumentos psicológicos enteramente culturales: primero es el lenguaje, la manera de contar, la escritura, las formas del arte.

3.- Es a través del aprendizaje que se desarrolla el pensamiento. Su noción de zona 
de desarrollo próximo encuentra una gran aceptación dentro de la Psicología del Aprendizaje.

Piaget es el fundador de la Teoría Constructivista de la Inteligencia; esta teoría se fue conociendo progresivamente en el curso del Siglo XX y ha tenido un gran impacto internacional. La fuerza innovadora de su teoría resulta de la combinación de tres orientaciones:

1.- Una orientación cognitiva: Piaget profundiza en la génesis de los procesos mentales que permiten el acceso a los conocimientos.

2.- Una visión estructuralista: la organización cognitiva se auto-estructura a través del conjunto de adquisiciones organizadas y ordenadas bajo la forma de estadío del desarrollo.

3.- Una teoría constructivista: Piaget supera las principales teorías de su época, por un lado al conductismo por el cual los conocimientos son totalmente adquiridos y por otro, a la teoría en la cual el desarrollo intelectual surge de la maduración del potencial genético del individuo (innatismo).

Para Piaget, la inteligencia se construye a través de la interacción entre el sujeto y su medio ambiente.

Según la teoría piagetiana, la inteligencia evoluciona por etapas, de un estadío a otro, de lo concreto a lo abstracto. Este modelo de desarrollo es a menudo comparado al ascenso de una escalera. De un estadío a otro, el pensamiento del niño cambia y sus razonamientos son a la vez, mejores y de otro tipo.

Sin embargo el arribo de las ciencias cognitivas y las nuevas técnicas de observación de niños hacia fines del Siglo XX, relanzaron las investigaciones sobre el desarrollo de la inteligencia, surgiendo de ese modo diversas escuelas:

- Los neopiagetianos: esta corriente intentó conciliar las teorías del desarrollo de
Piaget y la Psicología Cognitiva. En el inicio de los años 80 aparecieron las primeras alternativas serias a la teoría del desarrollo cognoscitivo de Piaget.

Ellos coinciden en que el desarrollo cognitivo se establece en estadíos, pero no acuerdan con la teoría de la escalera, por la cual el niño avanza de escalón en escalón sin posibilidades de retroceso ni avances más rápidos. Este modelo ha orientado las investigaciones hacia el intento de definir las características del razonamiento propio de cada estadío.

Algunos representantes de la corriente neopiagetiana son: Mounoud, de la Universidad de Ginebra, quien fue asistente de Piaget en el Centro Internacional de Epistemología Genética de Ginebra y Pascual-Leone de la Universidad de Toronto, considerado el padre de los neopiagetianos y autor de una teoría de operaciones constructivas (Pascual-Leone, 2000).

Case (1992) de la Universidad de Stanford, con su teoría de estructuras conceptuales centrales ha ejercido un fuerte impacto entre los neopiagetianos (Vaillè, 2009). Case fue uno de los primeros en conciliar la teoría piagetiana con la teoría cognitiva, intentando realizar una síntesis entre ambas. Este autor propuso un modelo en el que la memoria de trabajo es el elemento clave del desarrollo. La memoria de trabajo situada en el lóbulo frontal permite la realización de las operaciones mentales más complejas tales como planificación, cálculos, reflexión consciente, estrategia. Este tipo de memoria es solicitada al leer un texto. Ella combina las informaciones que provienen de la memoria sensorial (la visión de las palabras sobre una página con las informaciones ya guardadas en la memoria a largo plazo, el sentido de las palabras) para transformarlas, que es lo que permite deducir la significación del texto. Case comprobó que la utilización cada vez más eficiente de la memoria de trabajo es un elemento determinante del crecimiento cognitivo. Para este autor, la memoria de trabajo tal como es concebida, puede ser comparada a una red de clasificación donde el niño aprende, con la experiencia, a optimizar el uso de dicha red. Durante los 2 primeros 
años de vida, el niño no llega a clasificar 10 juguetes, pero en la medida que comprende, las clasificaciones son mejores. La capacidad de clasificación de la red no aumenta, pero la utilización de la misma se optimiza. Cuando este cambio se produce, dos factores estarían en la base de ello: el primero es la automatización. Case explica cómo ciertas tareas que devienen familiares, terminan por ser ejecutadas en forma mecánica, eso es lo que libera el espacio de almacenamiento en la memoria de trabajo. El otro factor es la maduración biológica. Las transiciones entre los estadíos estarían ligadas a un factor de maduración biológica y a los cambios en el nivel de la actividad eléctrica de las neuronas en el lóbulo frontal (Case, 1992). Este autor considera que la memoria de trabajo juega un rol decisivo en el desarrollo cognitivo.

Sin embargo, Case no rompe con la herencia piagetiana y conserva la hipótesis de un desarrollo en cuatro estadíos y sostiene la idea que el niño está provisto de modos de pensamiento específicos a ciertos tipos de conocimientos: el número, el espacio y la narración y adscribe al modelo de la escalera en su libro The mind's staircase (1992). Sin embargo, en los nuevos modelos dinámicos del desarrollo que hacen su aparición en los años 90, no se encuentra el concepto de progresión por estadío.

- Los evoluconistas: La mayoría de las teorías actuales están a favor de una progresión de la inteligencia gradual y cuasi continua, la inteligencia no evoluciona con saltos y hacia delante, sino con pequeños pasos marcados por períodos de mesetas y vuelta atrás de falsos pasos.

Dentro de esta concepción de la inteligencia se encuentran los trabajos de Siegler (2009), quien postula una teoría evolucionista del desarrollo. Siegler tomando el modelo de la biología en la evolución de las especies, comienza a trabajar sobre las contribuciones de la micro-evolución (como las mutaciones o las recombinaciones genéticas). Afirma Siegler:

"Es mucho más interesante, para comprender el desarrollo del niño, describir los microcambios que vienen de manera continua y gradual, más que intentar explicar los grandes cambios que se producen de un estadío del pensamiento al otro" (2009, p. 42).

El psicólogo Houdé (2009) comparte esta concepción evolucionista. El autor incorpora a la teoría piagetiana de asimilación y acomodación, dos conceptos nuevos: la activación e inhibición. Para el autor el proceso mental del pensamiento implica un estado de efervescencia constante, por eso lo asimila a una jungla. Es por esto que es fundamental la existencia de un mecanismo de bloqueo muy potente: la inhibición. Esta inhibición es parte del desarrollo y según Houdé, se da tanto en el pensamiento del niño como en el del adulto. Según Piaget la dinámica asimilación / acomodación conduce al cerebro humano desde las organizaciones sensorio-motrices y cognitivas cada vez más complejas: desde las acciones de los bebés hacia las operaciones lógicas y abstractas del adolescente y del adulto. Sin embargo, la dinámica asimilación / acomodación parece ser hoy en día insuficiente para describir cómo se desarrolla la inteligencia humana. Houdé propone adjuntar la activación / inhibición.

Desde los años 20, el joven Piaget ya inspirado por la visión de la Epistemología Biológica, no vio la importancia de la inhibición en el estudio del desarrollo cognitivo, cuando este concepto pluridisciplinario había sido introducido en Fisiología y en Psicología en el Siglo XIX y continuado en el Siglo XX por las escuelas de Sherrington, Pavlov y Freud. Esto es sin duda porque la inhibición era en el espíritu de Piaget muy negativo, significando represión, opuesto a la libertad para su teoría constructivista del desarrollo de la infancia. Esta incomprensión es ciertamente el error más importante de Piaget.

Houdé afirma que sus experiencias con imágenes cerebrales sobre el razonamiento lógico, le han permitido descubrir lo que ocurre en el cerebro de jóvenes adultos antes y después del aprendizaje de la inhibición de una estrategia perceptiva inadecuada, es decir antes y después de la corrección de un 
error de razonamiento. Se observa una neta configuración de redes cerebrales de la parte posterior del cerebro. El corte prefrontal es de la abstracción, de la lógica y del control cognitivo donde se encuentra la inhibición. Se observan errores perceptivos sistemáticos en ciertas tareas de lógica simple.

Aprender a inhibir la estrategia perceptiva inadecuada es también desarrollo, pues no es solamente construir y activar las estrategias cognitivas como lo pensaba Piaget, sino también aprender a inhibir las estrategias que entran en competencia dentro del cerebro. Por lo tanto, se puede afirmar que el desarrollo de la inteligencia no es lineal.

Esta corriente del pensamiento aborda el desarrollo cognitivo como una evolución en el sentido de Charles Darwin, integrando variación y selección; tiene una concepción dinámica de la inteligencia que evoluciona según ellos de manera gradual y caótica.

Algunos representantes de los evolucionistas son: Siegler de la Carnegie Mellon University de Pittsburg (EE.UU), quien compara el desarrollo cognitivo con olas que se superponen (2009); Houdé para quien el pensamiento está regulado por las capacidades de inhibición (2009) y Van Geert de Groningen de Holanda, que concibe el pensamiento como un sistema dinámico no lineal, que ilustra las curvas del desarrollo irregulares y caóticas (Houdé, 2009).

\section{ACTIVIDAD SENSORIO-MOTRIZ EN TANTO GÉNESIS DE LA INTELIGENCIA HUMANA}

Para el bebé piagetiano es necesario esperar la coordinación prensión - visión, que es cuando tiene la posibilidad de tomar un objeto que él ve para comprender el mundo de los objetos, de la misma manera que debe adquirir la locomoción autónoma para comprender la organización del espacio.

Además según Piaget, el bebé adquiere la conciencia de la permanencia de los objetos, es decir que los objetos continúan existiendo cuando ellos no son percibidos, a partir de una construcción lenta que se manifiesta alrededor de los 9 meses.
Sin embargo, las investigaciones de Fantz (1961) responden a las de Piaget, sobre un aspecto que este último no había considerado en toda su importancia: la percepción. Si bien esto no se contradice con la teoría piagetiana, el tema de la percepción permitió el ingreso de los bebés en los laboratorios. El método de la situación de la preferencia visual y la habituación - método que se utilizaba con cierto éxito en los animales, dio pie al desarrollo de nuevas conceptualizaciones en torno al tema del desarrollo cognitivo de los bebés.

En los inicios de la década de 1970 un método se pone a punto: la procedencia controlada del bebé. Consiste en observar la mirada del bebé colocado delante de un objeto. Según esta corriente de trabajo, cuando el bebé ve un objeto nuevo fija su mirada durante un cierto lapso de tiempo hasta que la duración de la fijación disminuye. Se considera que el bebé está habituado a ese objeto. Si el objeto que se le presenta es nuevo para él, él va a mirarlo largo tiempo. La variación y la duración de la mirada del bebé sobre el estímulo resulta ser un indicador de su capacidad para diferenciar lo que es nuevo de lo que no es y lo que es conocido de lo desconocido.

Sin embargo, es necesario diferenciar lo que los bebés saben de lo que saben hacer. La inteligencia es un proceso de acción sobre los objetos. La investigación que se informa colocó el énfasis en estudiar y evaluar la estrategia que realiza un bebé para resolver situaciones que se le presentan.

La EAIS no evalúa inteligencia emocional, ni se remite a un coeficiente intelectual de los infantes, sino que determina las estrategias que utiliza un bebé para resolver las situaciones que se le presentan, es decir, evalúa la inteligencia como un proceso.

\section{Características de los estadíos evaluados}

Siguiendo la teoría piagetiana, las características de los estadíos que se evalúan con la Escala Argentina de Inteligencia Sensoriomotriz son las siguientes: 


\section{EstADIOO III: ADAPTACIONES SENSORIO-MOTRICES IN- TENCIONALES}

Este estadío se desarrolla entre los 6 y 8 meses en los que ha progresado la postura y la prensión. El niño comienza a relacionar lo que percibe con lo que hace con sus manos. Los descubrimientos del niño son todavía realizados al azar, sin ningún objetivo preestablecido. La sola necesidad que está en juego sigue siendo la necesidad de la repetición. El niño repite los movimientos que han producido por azar una acción que le resulta interesante. El interés del niño recae sobre los resultados.

EstadíO IV: DISTINCIÓN ENTRE MEDIOS Y FINES. RESOLUCIÓN DE PROBLEMAS MEDIANTE ESTRATEGIAS CONOCIDAS

El IV estadío se desarrolla entre los 9 y 12 meses y marca un salto considerable en las adaptaciones de los bebés a nuevas situaciones. Por eso ya se puede hablar del inicio de un acto inteligente, porque el niño ya no se entregará a una simple repetición para reproducir resultados interesantes como en el estadío III, sino que ya distingue entre medios y fines, aplicando esquemas ya adquiridos a situaciones nuevas. Este estadío marca la transición hacia conductas más inteligentes porque el niño puede prever lo que puede suceder sin llegar todavía a la deducción. Por ejemplo, cuando el niño tira del mantel utilizando el mantel como medio para alcanzar un objeto que se encuentra lejos de su alcance.

\section{Estadí V: Descubrimiento de MEDIOS NUEVOS POR EXPERIMENTACIÓN ACTIVA}

Este estadío se desarrolla entre los 13 y 16 meses, es particularmente significativo en cuanto a la elaboración de formas más elevadas de la inteligencia empírica y coincide con el inicio de conductas instrumentales. Es el inicio de conductas activas de experimentación y descubrimiento de medios nuevos en vista de una solución de problemas nuevos.
Cuando el niño se enfrenta a problemas en los que su solución no es asimilable a esquemas habituales, va a descubrir nuevos medios, presentando una conducta imprevista. El resultado es siempre descubierto por tanteos y la experiencia comienza por una repetición de movimientos conocidos extraídos de otras situaciones. El niño va a producir una gran variedad de estrategias de una cierta originalidad en su conducta, es decir, aprende mediante ensayos activos.

\section{ESTADÍO VI: INVENCIÓN DE MEDIOS NUEVOS POR} COMBINACIÓN MENTAL (INSIGHT)

El VI estadío se desarrolla a partir de los 17 y hasta los 24 meses. El niño es capaz de encontrar los medios nuevos sin pasar por tanteos de ningún tipo. Resuelve situaciones nuevas sin tanteos, es decir, las resuelve por combinaciones interiorizadas, por una comprensión brusca de las situaciones o sea por insight. Hay mayor conocimiento de las relaciones entre los objetos por lo que puede resolver mentalmente las situaciones. Este estadío está caracterizado por la combinación mental de esquemas con posibilidad de resolución de ciertos problemas por deducción e invención de medios nuevos.

\section{OBJETIVOS}

Los objetivos del estudio que se informa fueron realizar una validación nacional de la Escala Argentina de Inteligencia Sensoriomotriz a fin de contar con percentiles nacionales para niños de 6 a 30 meses de edad e incorporar una nueva área del desarrollo en la evaluación de los niños menores de 3 años, el área del desarrollo cognitivo.

\section{Metodología \\ EQUIPO NACIONAL E INTERDISCIPLINARIO}

Para la realización de este trabajo se capacitó a profesionales de distintas regiones de nuestro país en el área del desarrollo cognos- 
citivo para niños pequeños para la administración de la EAIS y se organizaron servicios cognoscitivos para bebés en las diferentes provincias.

Se organizó así un grupo de 30 profesionales de diferentes provincias, de características verdaderamente interdisciplinarias, con experiencia en el trabajo en primera infancia, provenientes de diversas disciplinas: psicólogos, psicopedagogos, médicos fisiatras y pediatras, fonoaudiólogos, terapistas ocupacionales, estimuladores tempranos, profesionales en ciencias de la educación y psicomotricistas. Conformándose de esta manera, un verdadero equipo nacional e interdisciplinario (Oiberman, Santos, Mansilla, Dehollainz, Trucco, Menghi et al., 2010).

En la Tabla 1 se informan las regiones, provincias y ciudades de donde provienen los niños que conformaron la muestra como así también la frecuencia de niños evaluados en cada una de ellas.

\section{PARTICIPANTES}

Para conformar la muestra se establecieron los siguientes criterios de selección:

Se especificó un rango de edad (6 meses, 1 día - 29 meses, 30 días). Todos los niños debían obtener resultado de normalidad en la prueba de evaluación del desarrollo psicomotor (PRUNAPE - Lejarraga, Kelmansky, Pascucci \& Salamanca, 2004). Debían ser bebés de ambos sexos, argentinos y nacidos a término (edad gestacional igual o mayor a 37 semanas de gestación) y sin patología neonatal y sanos.

La población estuvo fundamentalmente compuesta por bebés de sectores medio-bajo, medio y medio-alto y un pequeño grupo de niños provenientes de sector bajo. Estos sectores socioculturales fueron determinados en base al nivel de educación de los padres. En la Tabla 2 se informan las características sociodemográficas de la muestra y el nivel de educación de los padres.

Los niños incluidos en la muestra fueron evaluados individualmente por un profesional capacitado, miembro del equipo de investi- gación, y las evaluaciones se realizaron con previa autorización firmada por los padres o adulto responsable.

\section{Procedimiento}

La tarea se realizó en cuatro etapas:

- En la primera etapa se hizo la convocatoria y la capacitación, así se conformó un equipo de 30 profesionales pertenecientes a diferentes provincias.

También se confeccionó un protocolo de investigación (ver Anexo 1), acordando los procedimientos y modalidades de la evaluación.

- En la segunda etapa se administró la EAIS, estableciéndose metas periódicas con el fin de lograr un desempeño semejante de los diferentes equipos de cada provincia. bés

- En la tercera etapa se evaluaron 800 be-

- En la cuarta etapa se elaboraron los percentiles nacionales.

La Tabla 2 informa las variables sociodemográficas de la muestra y fueron las siguientes: sexo, edad, peso de nacimiento, edad gestacional del niño, edad, nivel de educación y trabajo de los padres, número de hijo, situación de pareja, cuidado y concurrencia del niño al jardín y región de procedencia. En cada una de ellas y entre paréntesis se informa el número de casos de los cuales se obtuvo el dato correspondiente, ya que algunos padres no consignaron: edad, nivel de educación, trabajo, número de hijos, situación de pareja, cuidado y concurrencia del niño al jardín.

\section{INSTRUMENTOS}

La Escala Argentina de Inteligencia Sensorio-motriz (EAIS) está conformada por 45 pruebas en 4 situaciones diferentes:

- Serie A: Exploración de objetos.

- Serie B: Búsqueda del objeto desaparecido.

- Serie C: Intermediarios. Esta serie consta 
de 3 sub-series. C1: Utilización de la prolongación del objeto (cinta), C2: Utilización de la relación entre un objeto y su soporte y C3: Utilización de un instrumento (rastrillo).

- Serie D: Combinación de objetos. Consta de 2 sub-series: D1: Utilización de un instrumento para obtener un objeto del interior de un tubo (rastrillo-tubo) y D2: Combinación de objetos (tubo-cadena).

También se realizó una breve entrevista a los padres o acompañante responsable del bebé en la que se interrogó acerca de datos demográficos y algunos datos del niño y su familia (ver Anexo 2).

\section{TRATAMIENTO DE LOS DATOS}

La importancia de la utilización de la EAIS radica en que permite determinar si el bebé está en la etapa de crecimiento intelectual correcto o en su defecto, detectar precozmente retrasos o alteraciones en su inteligencia sensorio-motriz.

La conducta del niño denota una estrategia cognitiva para resolver la situación propuesta y así se le asigna un ítem en la serie y su correspondiente nivel de estadío. Luego de obtenido el estadío correspondiente en cada serie, se calcula la mediana (estadío resumen), la cual constituye su resultado final en la escala. Para valorar el nivel de estadío resumen alcanzado por cada niño se elaboró una nueva tabla de percentiles y tablas de percentiles por cada serie.

El estadío mediano se obtiene a partir de la mediana de todos los estadíos obtenidos en cada serie evaluada. Es decir, el niño alcanza un estadío por cada serie administrada. Estos estadíos se ordenan en forma creciente y se calcula la mediana correspondiente. Ese será el estadío mediano alcanzado por el niño.

La tabla de percentiles o baremo del estadío mediano se confeccionó a partir de dicho estadío alcanzado por cada uno de los 800 niños que conformaron la muestra (ver Tabla 3 ).

Esta tabla de percentiles provee una estimación del rango en el cual sería esperable ubicar a niños de 6 a 30 meses de edad. Los resultados que corresponden a un percentil 10 o menos se los considera de retraso. El resultado que corresponde a un percentil 25 se considera de riesgo y los percentiles iguales o mayores al 50 se consideran normal, es decir, esperable para la edad del niño.

\section{Resultados}

Se construyó una tabla de percentiles nacionales a partir de la muestra total de $800 \mathrm{ni}$ ños, con una edad mínima de 6 meses y 1 día y una edad máxima de 30 meses de edad cronológica. La Tabla 4 presenta las frecuencias según la edad de los niños.

Al analizar los percentiles de todos los niños correspondientes al nivel de estadío resumen obtenido y la valoración de los mismos, se obtuvieron los siguientes resultados:

El 72.25\% (578 niños) obtuvo resultados normales (percentil igual o mayor a 50), el $\mathrm{m} 14 \%$ (112 niños), percentiles de riesgo (percentil 25$)$ y el $13.75 \%$ (110 niños), percentiles de retraso (percentil 10 ó menos). Es decir, el $27.75 \%$ de los niños evaluados obtuvo resultados de riesgo y retraso en la inteligencia sensorio-motriz. Estos resultados son similares a los observados en investigaciones anteriores referentes al proceso de inteligencia sensorio-motriz (Oiberman, 2009).

Se observaron diferencias en la edad en la que los bebés alcanzan los diferentes estadíos. De 169 bebés que inician el V estadío, el $67 \%$ (113) lo hacen antes de los 13 meses (ver Tabla 5) y no a los 13 meses como lo planteaba Piaget.

Al haber duplicado la muestra de bebés no fue necesario agruparlos por edades hasta los 25 meses, lo cual permite contar con un grupo de referencia por cada mes desde los 6 hasta los 25 meses. Se agruparon las siguientes edades: 26 y 27 y $28-29$ y 30 meses (ver Tabla 6).

Dado que en la investigación realizada en el año 2002 (Oiberman, Mansilla \& Orellana, 2002) solo el $20 \%$ de 323 bebés de 24 meses de edad finalizaban el VI Estadío, se consideró importante investigar a qué edad verda- 
deramente los niños finalizan el período sensorio-motriz en nuestro país.

En el estudio que se informa se comprobó que el VI Estadío finaliza a los 30 meses: el $75 \%$ de los niños de entre $28-29$ y 30 meses se localizan en el final del VI Estadío (ver Tabla 6).

Sintetizando, en la muestra estudiada los bebés no finalizan el período sensorio-motor a los 24 meses tal como lo planteaba Piaget. Según los trabajos de Piaget y colaboradores a partir de los 17 meses en adelante, el desarrollo cognoscitivo del niño se debe localizar en el Estadío VI y finalizando el mismo a los 24 meses de edad (Piaget, 1973). El VI Estadío está caracterizado por la combinación mental de esquemas con posibilidad de resolución de ciertos problemas por deducción, invención de medios nuevos y comienzo de evocaciones representativas.

\section{Discusión}

Esta investigación permitió conocer más profundamente las etapas del proceso cognoscitivo y reafirmar que su desarrollo debe ser valorado como un área especifica y diferenciada en la evaluación del desarrollo psicomotor de los niños.

Esta nueva área constituye junto a las áreas de motricidad, coordinación, lenguaje y social, la quinta área del desarrollo del niño. La incorporación de dicha área en la evaluación del desarrollo de los bebés argentinos hizo posible conocer más completa e integralmente, cada etapa de los procesos intelectuales comprendidos entre los 6 y 30 meses, en las diferentes poblaciones de bebés de Argentina.

En las tablas de percentiles de la muestra estudiada se observa tal como lo planteaba Piaget, que el VI Estadío es transitado a partir de los 18 meses.

Se comprueba que el inicio de la representación mental se logra en los bebés argentinos también a los 18 meses aproximadamente.

Sin embargo, se observa un adelanto de los bebés en el acceso al V Estadío al comparar los resultados obtenidos con los propuestos por Piaget hace 40 años.
En efecto, se observó una tendencia de acceso a estadíos superiores en edades más tempranas en esta nueva población. Por otra parte, los resultados revelan que es frecuente encontrar niños en etapas de transición de un estadío a otro, por ejemplo: Fin V - Inicio VI. Los niños pueden estar entre finales de un estadío e iniciar el otro. Ello también puede atribuirse al estudio minucioso que permitió profundizar los procesos del recorrido de la inteligencia de los bebés y evaluar más finamente la ubicación de finales de un estadío e inicios del siguiente.

Se encontró una progresión de la inteligencia gradual y cuasi continua: ella no evoluciona por saltos y hacia delante como en el modelo escalera (Houdé, 2009), sino por pequeños pasos marcados por períodos de mesetas y vuelta atrás de falsos pasos. Esto se observa a los 19 meses que es cuando el niño alcanza un estadío más avanzado y a los 21 , 22 y 23 meses hay un retroceso. Esto se comprueba no solo al analizar los percentiles para el estadío mediano sino que también se repite la misma situación en todas las series de la prueba.

Esto concuerda con los nuevos modelos dinámicos del desarrollo que hacen su aparición en los años 90 y cuestiona el concepto de progresión por estadío. Dentro de esta concepción de la inteligencia se encuentran los trabajos de Siegler (2009), quien postula una teoría evolucionista del desarrollo.

Estos descubrimientos se asocian también a los estudios de algunos neopiagetianos, quienes, tomando el modelo de la biología en la evolución de las especies, comienzan a trabajar sobre las contribuciones de la micro-evolución (como las mutaciones o las recombinaciones genéticas).

Por otra parte, comparando con los resultados obtenidos en la validación original de 2002, a partir de esta muestra de validación nacional de 2012 se ha logrado un análisis más minucioso del recorrido de los niños por cada estadío. Se ha logrado establecer con mayor rigurosidad el inicio, el transcurrir y la finalización de cada estadío, considerando que es más eficaz detectar el proceso gradual por el cual un niño accede a un nuevo ni- 
vel de procesamiento de la realidad circundante y comprender y describir los microcambios, pues ello nos permite un mejor estudio individual de cada bebé.

Se comprobó que en las micro-evoluciones que realizan los bebés, y que son un aporte a la teoría del desarrollo psicomotor de los niños, ellos pasan por los subestadíos propuestos por la escuela piagetiana hace 40 años.
Sin embargo, si para Piaget los cambios se producían de a saltos, siguiendo descriptivamente una escalera, los resultados obtenidos llevan a considerar que el crecimiento del desarrollo intelectual de los bebés se asemeja más a una rampa, ya que es posible observar pequeños retrocesos y avances, y el desarrollo de la inteligencia es cuasicontinuo.

TABLA 1

REGIONES, PROVINCIAS Y CIUDADES PARTICIPANTES

\begin{tabular}{|c|c|c|}
\hline Región & Provincia & Ciudad \\
\hline $\begin{array}{l}\text { Metropolitana } \\
219 \text { niños }(27.4 \%)\end{array}$ & Buenos Aires: 219 niños & $\begin{array}{l}\text { Ciudad Aut. de Buenos Aires } \\
\text { Mar del Plata } \\
\text { Bahía Blanca } \\
\text { Tandil }\end{array}$ \\
\hline $\begin{array}{l}\text { Centro } \\
205 \text { niños }(25.6 \%)\end{array}$ & $\begin{array}{l}\text { Córdoba: } 29 \text { niños } \\
\text { Entre Ríos: } 91 \text { niños } \\
\text { Santa Fe: } 85 \text { niños }\end{array}$ & $\begin{array}{l}\text { Córdoba } \\
\text { Ciudad de mis sueños } \\
\text { Paraná } \\
\text { Viale } \\
\text { Libertador Gral. San Martín } \\
\text { Sunchales } \\
\text { San Carlos }\end{array}$ \\
\hline $\begin{array}{l}\text { Norte Grande Argentino } \\
77 \text { niños }(9.6 \%)\end{array}$ & $\begin{array}{l}\text { Salta: } 50 \text { niños } \\
\text { Chaco: } 12 \text { niños } \\
\text { Misiones: } 15 \text { niños }\end{array}$ & $\begin{array}{l}\text { General Mosconi } \\
\text { Resistencia } \\
\text { Las Palmas } \\
\text { Roque Sáenz Peña } \\
\text { Posadas }\end{array}$ \\
\hline $\begin{array}{l}\text { Nuevo Cuyo } \\
25 \text { niños (3.1\%) }\end{array}$ & Mendoza: 25 niños & $\begin{array}{l}\text { Maipú } \\
\text { Godoy Cruz } \\
\text { Guaymallén }\end{array}$ \\
\hline $\begin{array}{l}\text { Patagónica } \\
274 \text { niños (34.2\%) }\end{array}$ & $\begin{array}{l}\text { Río Negro: } 104 \text { niños } \\
\text { Santa Cruz: } 170 \text { niños }\end{array}$ & $\begin{array}{l}\text { Cipoletti } \\
\text { Allen } \\
\text { Bariloche } \\
\text { Río Gallegos }\end{array}$ \\
\hline
\end{tabular}


TABLA 2

CARACTERISTICAS SOCIODEMOGRÁFICAS DE LA MUESTRA

\begin{tabular}{|c|c|}
\hline $\begin{array}{l}\text { Sexo RN - } n(\%) \\
\text { Femenino } \\
\text { Masculino }\end{array}$ & $\begin{array}{l}364(45.5) \\
436(54.5)\end{array}$ \\
\hline $\begin{array}{l}\text { Peso RN }(n) \\
M \pm D E \\
\text { Mín - Max }\end{array}$ & $\begin{array}{c}3350.3 \pm 447.9 \\
1996-4900\end{array}$ \\
\hline $\begin{array}{l}\text { Edad gestacional }(n) \\
M \pm D E \\
\text { Mín - Max }\end{array}$ & $\begin{array}{c}39.1 \pm 1.1 \\
37-42\end{array}$ \\
\hline $\begin{array}{l}\text { Edad del niño al momento de la evaluación }(n) \\
M \pm D E \\
\text { Mín - Max } \\
\text { Franjas etarias } n(\%) \\
6-12 \text { meses } \\
13-18 \text { meses } \\
19-24 \text { meses } \\
25-30 \text { meses }\end{array}$ & $\begin{array}{c}15.7 \pm 6.5 \\
6-30 \\
\\
307(38.4) \\
222(27.7) \\
173(21.6) \\
98(12.2)\end{array}$ \\
\hline $\begin{array}{l}\text { Edad de los padres }(M \pm D E) \\
\text { Edad materna }(n=786) \\
\text { Edad paterna }(n=764)\end{array}$ & $\begin{array}{l}28.6 \pm 6.3 \\
31.5 \pm 7.3\end{array}$ \\
\hline $\begin{array}{l}\text { Edad por categorías } n(\%) \\
\text { Edad materna }(n=786) \\
13-17 \text { años } \\
18-25 \text { años } \\
26-34 \text { años } \\
35-50 \text { años }\end{array}$ & $\begin{array}{c}20(2.5) \\
249(31.7) \\
375(47.7) \\
142(18.1)\end{array}$ \\
\hline $\begin{array}{l}\text { Educación } n(\%) \\
\text { Educación materna }(n=752) \\
\text { Primario Incompleto } \\
\text { Primario Completo - Secundario Incompleto } \\
\text { Sec. Completo - Terciario - Univ. Incompleto } \\
\text { Terciario o Universitario Completo }\end{array}$ & $\begin{array}{c}29(3.9) \\
241(32.0) \\
265(35.2) \\
217(28.9)\end{array}$ \\
\hline
\end{tabular}

(Continúa) 
TABLA 2 (CONTINUACIÓN)

CARACTERÍSTICAS SOCIODEMOGRÁFICAS DE LA MUESTRA

\begin{tabular}{|c|c|}
\hline $\begin{array}{l}\text { Educación paterna }(n=715) \\
\text { Primario Incompleto } \\
\text { Primario Completo - Secundario Incompleto } \\
\text { Sec. Completo - Terciario - Univ. Incompleto } \\
\text { Terciario o Universitario Completo }\end{array}$ & $\begin{array}{c}36(5.0) \\
232(32.5) \\
279(39.0) \\
168(23.5)\end{array}$ \\
\hline $\begin{array}{l}\text { Trabajo } n(\%) \\
\text { Trabajo materno }(n=779) \\
\text { Trabajo paterno }(n=742)\end{array}$ & $\begin{array}{l}411(52.8) \\
687(92.6)\end{array}$ \\
\hline $\begin{array}{l}\text { Número de hijo } \\
\text { Mín - Max } \\
M \pm D E\end{array}$ & $\begin{array}{c}1-10 \\
1.9 \pm 1.3\end{array}$ \\
\hline $\begin{array}{l}\text { Número de hijos } n(\%)(n=788) \\
1 \text { hijo } \\
2-3 \text { hijos } \\
\text { Más de } 3 \text { hijos }\end{array}$ & $\begin{array}{l}417(52.9) \\
291(36.9) \\
80(10.2)\end{array}$ \\
\hline $\begin{array}{l}\text { Situación de pareja } n(\%)(n=729) \\
\text { Pareja estable } \\
\text { Pareja inestable } \\
\text { Madre sola con apoyo familiar } \\
\text { Madre sola sin apoyo familiar }\end{array}$ & $\begin{aligned} 635 & (87.1) \\
18 & (2.5) \\
56 & (7.7) \\
20 & (2.7)\end{aligned}$ \\
\hline $\begin{array}{l}\text { Cuidado del niño } n(\%)(n=684) \\
\text { Familiares } \\
\text { Otros } \\
\text { Ambos }\end{array}$ & $\begin{array}{l}577(84.4) \\
57(8.3) \\
50(7.3)\end{array}$ \\
\hline Concurrencia del niño al jardín $n(\%)(n=691)$ & $216(31.3)$ \\
\hline $\begin{array}{l}\text { Regiones } \\
\text { Metropolitana (CABA, Bs. As.) } \\
\text { Centro (Córdoba, Santa Fe, Entre Ríos) } \\
\text { Norte Grande Argentino (Salta, Misiones, Chaco) } \\
\text { Nuevo Cuyo (Mendoza) } \\
\text { Patagónica (Río Negro, Santa Cruz) }\end{array}$ & $\begin{array}{l}219(27.4) \\
205(25.6) \\
77(9.6) \\
25(3.1) \\
274(34.2)\end{array}$ \\
\hline
\end{tabular}




\begin{tabular}{|c|c|c|c|}
\hline & $=$ & 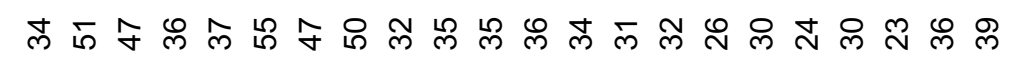 & $\stackrel{\infty}{\circ}$ \\
\hline \multirow{8}{*}{ 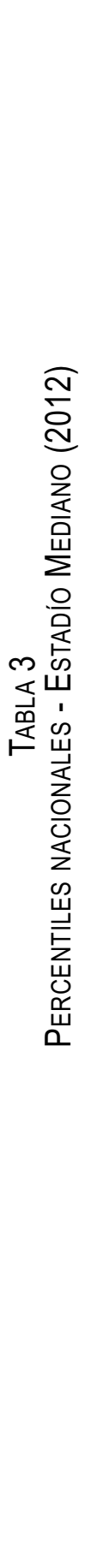 } & $\sum^{\frac{x}{\pi}}$ & 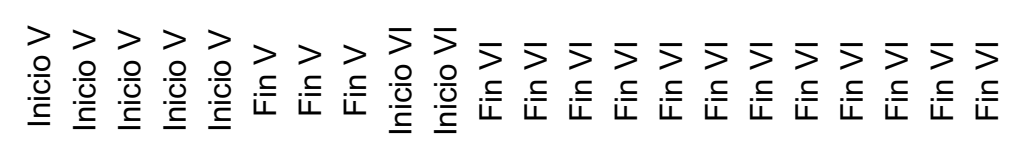 & \\
\hline & 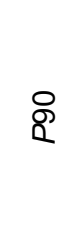 & 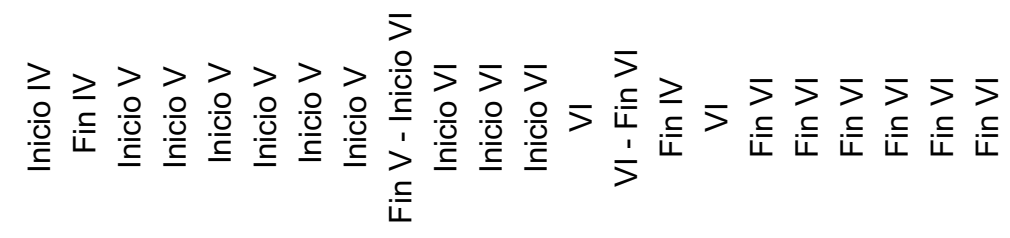 & \\
\hline & $\stackrel{n}{a}$ & 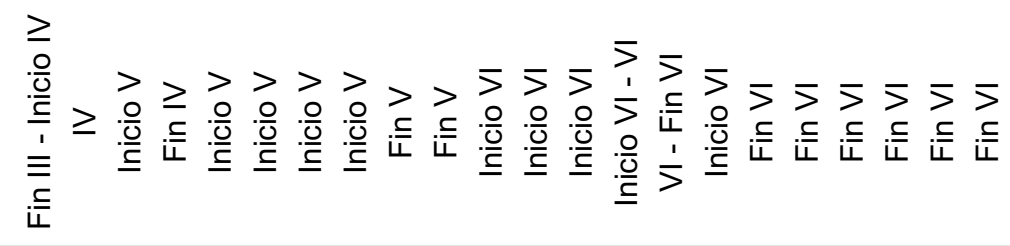 & \\
\hline & 员 & 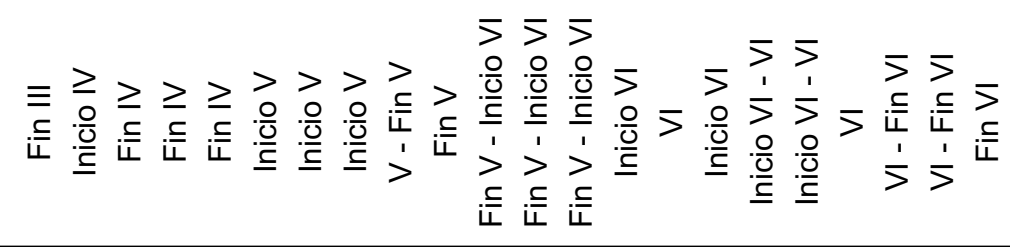 & \\
\hline & $\stackrel{\mathscr{Q}}{\mathrm{Q}}$ & 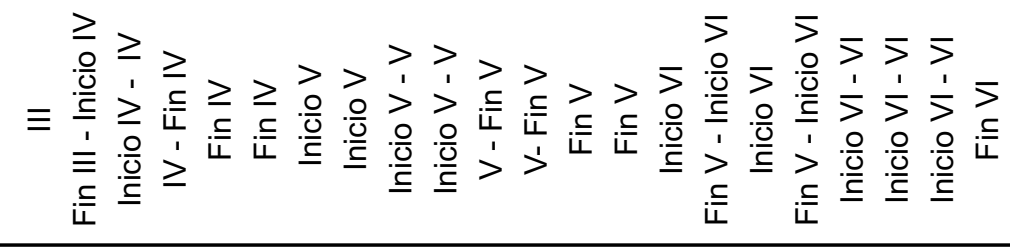 & \\
\hline & $\stackrel{\circ}{2}$ & 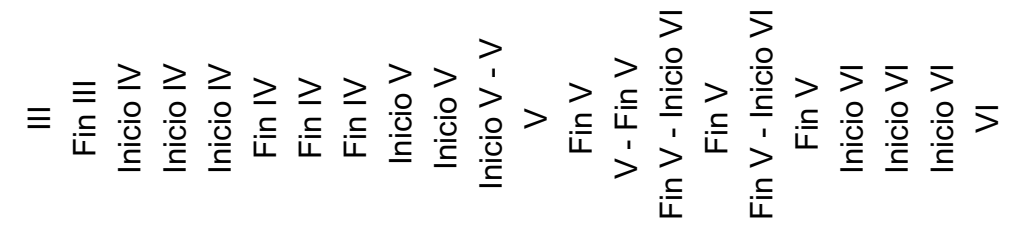 & \\
\hline & 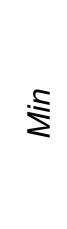 & 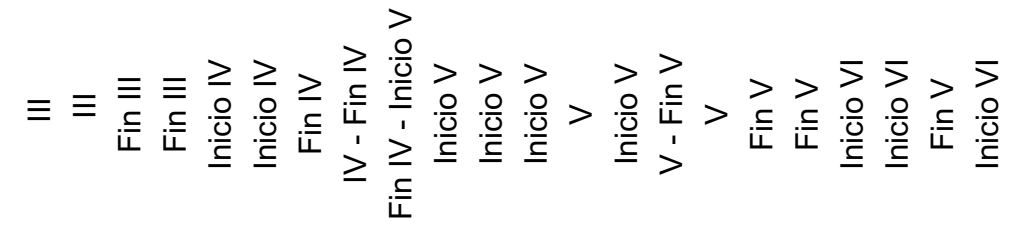 & \\
\hline & 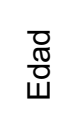 & 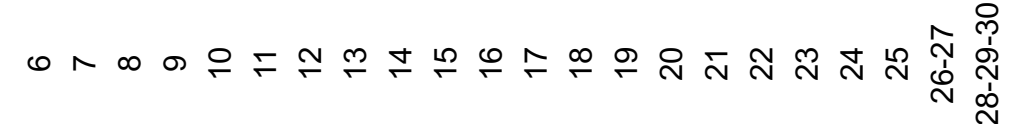 & $\begin{array}{l}\overline{\widetilde{\sigma}} \\
\stackrel{0}{\circ}\end{array}$ \\
\hline
\end{tabular}


Percentiles nacionales (EAIS)

TABLA 4

FRECUENCIA Y PORCENTAJE DE NIÑOS EVALUADOS POR EDAD

\begin{tabular}{|c|c|c|}
\hline Edad en meses & $n$ & $\%$ \\
\hline 6 & 34 & 4.25 \\
7 & 51 & 6.38 \\
8 & 47 & 5.88 \\
9 & 36 & 4.50 \\
10 & 37 & 4.63 \\
11 & 55 & 6.88 \\
12 & 47 & 5.88 \\
13 & 50 & 6.25 \\
14 & 32 & 4.00 \\
15 & 35 & 4.38 \\
16 & 35 & 4.38 \\
17 & 36 & 4.50 \\
18 & 34 & 4.25 \\
19 & 31 & 3.88 \\
20 & 32 & 4.00 \\
21 & 26 & 3.25 \\
22 & 30 & 3.75 \\
23 & 24 & 3.00 \\
24 & 30 & 3.75 \\
25 & 23 & 2.88 \\
$26-27$ & 36 & 4.50 \\
$28-29-30$ & 39 & 4.88 \\
& & \\
\hline Total & 800 & 100 \\
\hline
\end{tabular}


TABLA 5

NIÑOS QUE INICIAN EL V ESTADíO

\begin{tabular}{|c|c|c|c|}
\hline Edad en meses & $n$ & $\%$ & \% Acumulado \\
\hline & 1 & .59 & .59 \\
6 & 2 & 1.18 & 1.77 \\
7 & 13 & 7.69 & 9.46 \\
8 & 7 & 4.14 & 13.06 \\
9 & 17 & 10.06 & 23.66 \\
10 & 35 & 20.71 & 44.37 \\
11 & 38 & 22.50 & 66.87 \\
12 & 41 & 24.26 & 91.13 \\
13 & 5 & 2.96 & 94.09 \\
14 & 5 & 2.96 & 97.05 \\
15 & 2 & 1.18 & 98.23 \\
16 & 2 & 1.18 & 99.41 \\
17 & 1 & .59 & 100 \\
19 & 169 & 100 & \\
\hline Total & & & \\
\hline
\end{tabular}

TABLA 6

NiÑOS QUE FINALIZAN EL VI Estadío

\begin{tabular}{|c|c|c|c|}
\hline Edad en meses & $n$ & $\%$ & $\%$ Acumulado \\
\hline 16 & 1 & 1.09 & 1.09 \\
17 & 2 & 2.17 & 3.26 \\
18 & 1 & 1.09 & 4.35 \\
19 & 3 & 3.26 & 7.61 \\
20 & 7 & 7.61 & 15.22 \\
21 & 1 & 1.09 & 16.31 \\
22 & 8 & 8.70 & 25.01 \\
23 & 7 & 7.61 & 32.62 \\
24 & 10 & 10.87 & 43.49 \\
25 & 10 & 10.87 & 54.36 \\
26 y 27 & 12 & 13.04 & 67.04 \\
28,29 y 30 & 30 & 32.60 & 100 \\
\hline Total & 92 & 100 & \\
\hline
\end{tabular}


ANEXO 1

Protocolo de inVEStigación de LA EVALUACIÓn NACIONAL CON LA Escala ARgentina de INTELIGENCIA SENSORIO-MOTRIZ (EAIS)

Nombre:

Fecha de evaluación:

Fecha de nacimiento: __ /

Apellido: días

\begin{tabular}{|l|l|l|l|l|}
\hline Series & Pruebas & Item & Estadíos & Percentil \\
\hline & Exploración del objeto & & & \\
Búsqueda del objeto desaparecido & $\begin{array}{l}\text { 1. Cinta } \\
\text { 2. Soporte } \\
\text { 3. Instrumento }\end{array}$ & & \\
1. Tubo - Rastrillo & 2. Tubo - Cadena & & & \\
& &
\end{tabular}

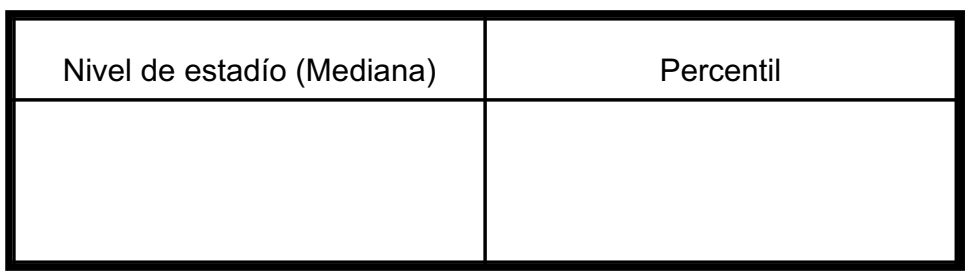

PRUNAPE

\begin{tabular}{|c|c|}
\hline Normal & \\
Sí & \\
No & \\
\hline
\end{tabular}


ANEXO 2

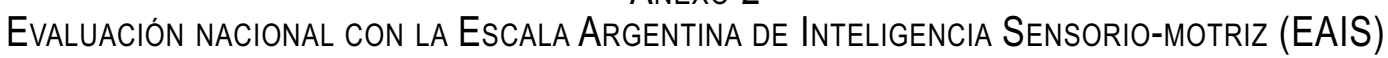

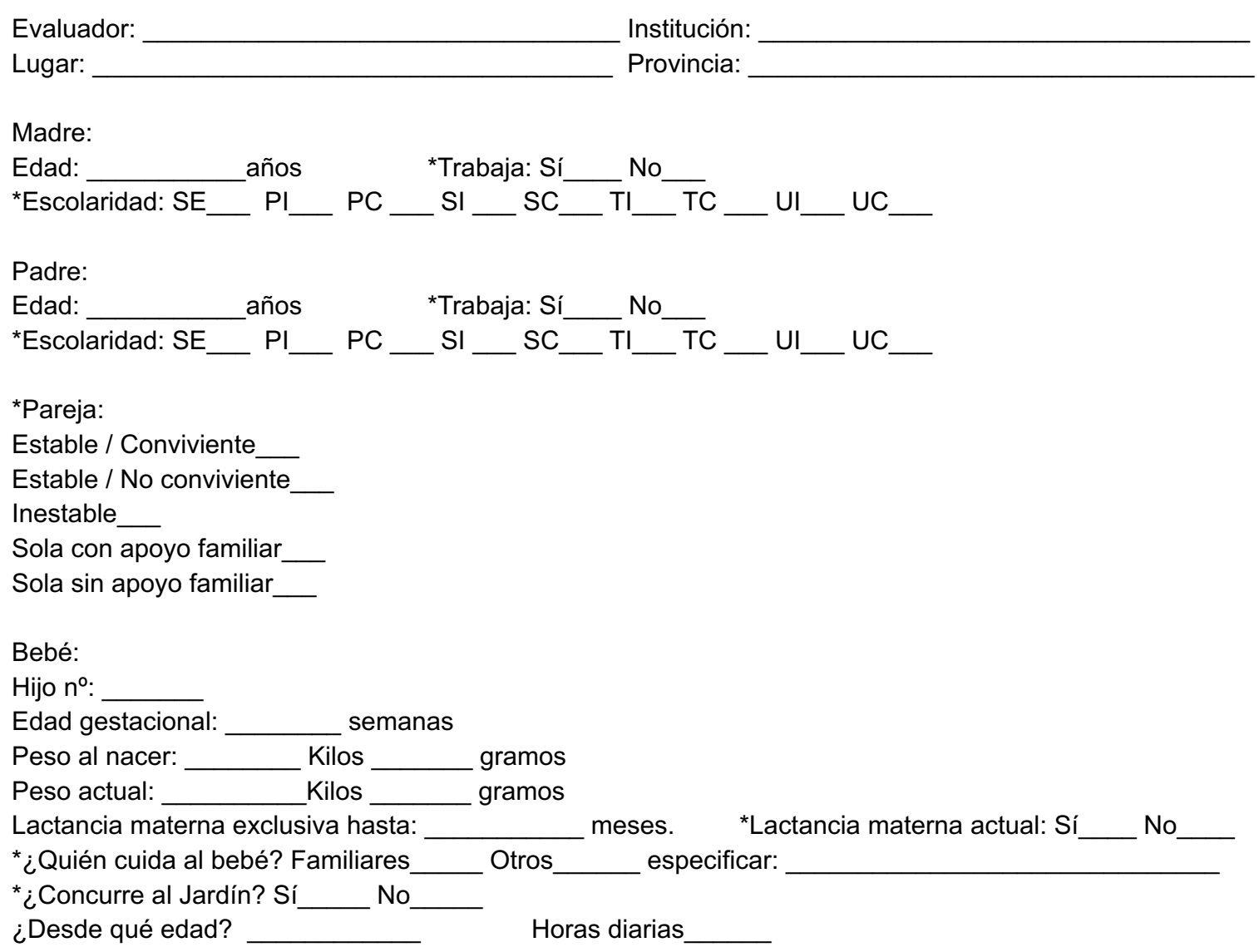

Observaciones - Otra información:

*Marcar con una cruz la respuesta que corresponda. 


\section{REFERENCIAS BIBLIOGRÁFICAS}

Casati, I. \& Lezine, I. (1969). Les étapes de l'intelligence sensori-motrice [Stages of sensor motor intelligence]. Paris: Centre Psychologie Appliqué.

Case, R. (1992). The mind's staircase: Exploring the conceptual underpinnings of children's thought and knowledge. Hillsdale NJ: Erlbaum.

Fantz, R.L. (1961). The origin of form perception. Scientific American, 204(5), 66-72. doi: 10.1038 .

Houdé, O. (2009). Piaget, quarante ans après. En M. Fournier \& R. Lécuyer (Coords.), L'intelligence de l'enfant [The intelligence of infant]. Paris: Sciences Humaines Editions.

Lejarraga, H., Kelmansky, D., Pascucci, M.C. \& Salamanca, G. (2004). Prueba Nacional de Pesquisa. PRUNAPE (Manual técnico) [National Research Test. Technical manual]. Buenos Aires: Fundación Hospital de Pediatría Dr. Juan P. Garraham.

Oiberman, A. (2009). Del laboratorio a la asistencia: Instalación de una cámara Gesell para evaluar el nivel cognoscitivo alcanzado en niños pequeños [From laboratory to assistance: Installing a Gesell chamber to assess the cognitive level reached in young children]. Investigaciones en Psicología, 14(13), 77-91.

Oiberman, A., Mansilla, M. \& Orellana, L. (2002). Nacer y pensar [Be born and think]. Buenos Aires: CIIPME - CONICET.

Oiberman, A., Orellana, L. \& Mansilla, M. (2006). Evaluación de la inteligencia en bebés argentinos: Escala Argentina de Inteligencia
Sensorio-motriz [Assessment of intelligence in Argentine babies: Argentine Scale of Sensor motor Intelligence]. Revista Archivos Argentinos de Pediatría, 104(4), 316-325.

Oiberman, A.J., Santos, M.S., Mansilla, M., Dehollainz, I., Trucco, M.A., Menghi, M.S., et al. (2010). Resultados preliminares de 7 provincias argentinas en el marco de una propuesta de evaluación nacional de la inteligencia sensorio-motriz a bebés de 6 a 30 meses [Preliminary results of seven argentinian provinces under a national assessment of sensorimotor intelligence to infants from 6 to 30 months old]. Anuario de Investigaciones, 17, 213-227.

Pascual-Leone, J. (2000). Reflections on working memory: Are the two models complementary? Journal of Experimental and Child Psychology, 77, 138-154. doi:10.1006.

Piaget, J. (1973). El nacimiento de la inteligencia en el niño [The birth of intelligence in infant]. Ginebra: Neuschatêl, Delachaux et Niestlé.

Siegler, R. (2009). À chaque âge son mode de pensée? En M. Fournier \& R. Lécuyer (Coords.), L'intelligence de l'enfant [The intelligence of infant] (pp. 34-42). Paris: Sciences Humaines Editions.

Vaillè, H. (2009) Jean Piaget et les stades de l'íntelligence. En M. Fournier \& R. Lécuyer (Coords.), L'intelligence de l' enfant [The intelligence of the infant] (pp. 25-33). Paris: Sciences Humaines Editions.

Vygotsky, L. (1995). Pensamiento y lenguaje [Thought and language]. Buenos Aires: Paidós.

Wallon, H. (1974). Del acto al pensamiento [Act of thinking]. Buenos Aires: Editorial Psiqué.

Centro Interdisciplinario de Investigaciones en Psicología Matemática y Experimental "Dr. Horacio J. A. Rimoldi" (CIIPME) Consejo Nacional de Investigaciones Científicas y Técnicas (CONICET)

Ciudad Autónoma de Buenos Aires - República Argentina

Fecha de recepción: 7 de mayo de 2012 Fecha de aceptación: 15 de noviembre de 2012 
